\title{
A Convenient Conversion Chart for Obtaining Serum LDL-Cholesterol Values
}

\author{
NOBUMITSU ISHIHARA \\ Ishihara Surgical Clinic, 427-4 Miyanojin Goroumaru, \\ Kurume 830, Japan
}

Received for publication February 15, 1995

\begin{abstract}
Summary: I devised a convenient conversion chart to obtain the LDLcholesterol value from the measured total cholesterol, HDL-cholesterol and triglyceride values without calculations. The principles and the practical use of the chart are described. Its application to the development of slide calculators that allow direct reading of LDL-cholesterol value is also discussed.
\end{abstract}

Key words: serum total cholesterol - HDL-cholesterol - triglyceride LDL-cholesterol - hyperlipemia

\section{Introduction}

The important role of LDL-cholesterol in fat metabolism has recently become well recognized. A serum LDLcholesterol value greater than $150 \mathrm{mg} / \mathrm{dl}$ is considered to be abnormal. To calculate the serum LDL-cholesterol value, equations (1) and (2) are presently in use, corresponding to serum triglyceride values greater or less than $400 \mathrm{mg} / \mathrm{dl}$, respectively (Nakamura, 1987).

[LDL-cholesterol] $=[$ Total cholesterol $]-$ [HDL-cholesterol] $-0.2 \times$ [triglyceride]

[LDL-cholesterol $]=[$ Total cholesterol $]-$ [HDL-cholesterol] $-0.16 \times$ [triglyceride]

I attempted visualization of these equations and devised a simple and easy conversion chart which enables one to obtain the LDL-cholesterol value graphi- cally. Here I will describe the principles and the practical use of the chart and also discuss its application to development of slide rule-type and slide disctype calculators that allow direct reading of LDL-cholesterol values.

\section{Laboratory Data}

The following laboratory data were adopted as the standard values (mg/dl): 122-255 for total cholesterol, $43 \pm 10$ (male) and $56 \pm 14$ (female) for HDL-cholesterol, $30-135$ for triglyceride, and $121.9 \pm 24.2$ for LDL-cholesterol (Kawai et al. 1987). Their actual varying ranges are assumed to be 40-600 for total cholesterol, 10-130 for HDL-cholesterol and 30-1000 for triglyceride.

\section{Principles}

Consider the first quadrant of an $x-y$ plane in which total cholesterol and HDL-cholesterol values are plotted on the $\mathrm{y}$ - and $\mathrm{x}$-axis with the same scale 
units, respectively (Fig. 1). A line parallel to the $\mathrm{x}$-axis is drawn from a measured total cholesterol point on the $y$ axis and another line parallel to the $y$ axis is drawn from a measured HDLcholesterol point on the $\mathrm{x}$-axis. If we define $\mathrm{A}$ as the difference obtained by subtracting the HDL-cholesterol value (X) from the total cholesterol value $(Y)$, i.e., $\mathrm{A}=\mathrm{Y}$-X, then $\mathrm{A}$ is the $\mathrm{y}$-intercept of a line with a slope of unity, which passes through the intersection point of the two

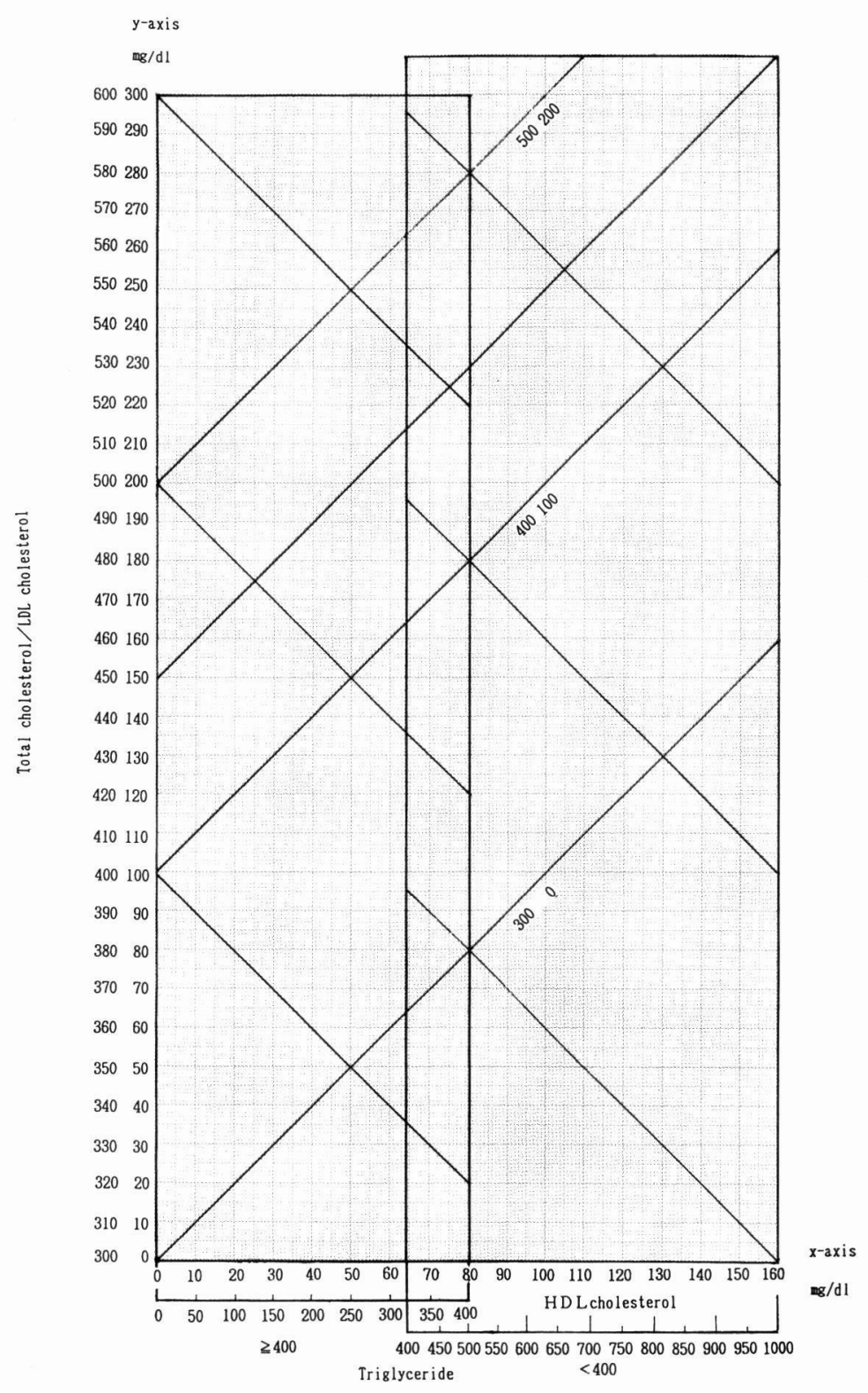

Fig. 1. Conversion chart for LDL-cholesterol. 
lines perpendicular to each other.

Next, in addition to the HDL-cholesterol scale, put two scales for triglyceride on the y-axis: the left scale is used when the measured triglyceride value is less than $400 \mathrm{mg} / \mathrm{ml}$, and the right scale is used when the triglyceride value is $400 \mathrm{mg} / \mathrm{ml}$ or above. The products, i.e., the triglyceride values multiplied by 0.2 or 0.16 , respectively, are directly read on the HDL-cholesterol scale. Because of the different multiplying factors used with the triglyceride values ( 0.2 and 0.16 ), an overlapping region appears between the two triglyceride scales. In the case of triglyceride values of less than $400 \mathrm{mg} / \mathrm{ml}$, select the range of 0 to $80 \mathrm{on}$ the HDL-cholesterol scale, and when these values are $400 \mathrm{mg} / \mathrm{ml}$ or more, use the range from 64 and above. Again a line parallel to the $\mathrm{x}$-axis is drawn from $\mathrm{A}$ and another line parallel to the $\mathrm{y}$-axis is drawn from the measured triglyceride point on the selected triglyceride scale. Find the intersection point of the two lines. The LDL-cholesterol value in question is the y-intercept of a line with a slope of unity, which passes through this intersection point.

\section{Practical Use}

1) Draw a line perpendicular to the $y$-axis from a point corresponding to the measured total cholesterol value on the $\mathrm{y}$-axis.

2) Draw a line perpendicular to the $\mathrm{x}$-axis from a point corresponding to the measured HDL-cholesterol value on the $\mathrm{x}$-axis.

3) Draw a line with an angle of $45^{\circ}$ through the point where the two lines intersect, and obtain its y-intercept.

4) Draw a line perpendicular to the $y$-axis from the intercept.

5) Select the appropriate triglyceride scale according to the measured triglyceride value. Draw a line perpendicular to the $\mathrm{x}$-axis from a point corresponding to the triglyceride value on the $\mathrm{x}$-axis.

6) Draw a line with an angle of $45^{\circ}$ through the intersection point of the two lines drawn in 4) and 5), and obtain its yintercept. Read the coordinate of the intercept, representing the LDL-cholesterol value.

\section{Slide Rule and Slide Disc Calculator}

Slide rule calculator: Graduate the total cholesterol scale on a ruler (ruler 1) and HDL-cholesterol and triglyceride scales on another ruler (ruler 2) as in the conversion chart. Put the two rulers face to face with each other in such a way that the respective cholesterol scales run in the same direction.

When the measured total cholesterol value on the ruler 1 is adjusted to the measured HDL-cholesterol value on the ruler 2 , the reading on ruler 1 corresponding to the origin (zero) of ruler 2 represents the difference between total cholesterol and HDL-cholesterol, i.e., total cholesterol minus HDL-cholesterol. Next, adjust the measured triglyceride value on ruler 2 to the difference value obtained on ruler 1 . Then the reading on ruler 1 corresponding to the origin (zero) of ruler 2 is LDL-cholesterol value in question.

In order to perform the above operations precisely, attach a transparent cursor with a line running perpendicular to the cholesterol scales over the two rulers; the rulers must be able to slide smoothly under the cursor.

Slide disc calculator: The principles are 
the same as those with the slide rule; however, it consists of, instead of rulers, a larger and a smaller disc and a transparent disc cursor with a line indicating the diameter. These discs are concentrically put together from bottom to top in order of the larger disc, the smaller disc and the cursor. They must freely turn around the central axis.

Graduate the total cholesterol scale on the larger disc and HDL-cholesterol and triglyceride scales on the smaller disc in the same direction as in the conversion chart. Assuming that the larger and smaller discs are the respective counterparts of the slide rule calculator, ruler 1 and 2, the operation is achieved in a manner quite similar to that with the slide rule calculator.

\section{Conclusions}

When the values of total cholesterol, HDL-cholesterol and triglyceride in serum are known from blood analyses, one can readily obtain serum LDL-cholesterol values from the convenient conversion chart using a pencil and a triangle.

Acknowledgments: The author would like to thank Prof. M. Noguchi for critical reading of the manuscript and Ms. M. Kitajima for secretarial arts.

\section{References}

Kawal, T., Niwa, M. and Hashimoto, S. (1987). The standard values of laboratory data. Nihon Ishikai Zasshi 98 (Suppl), 314-352. (in Japanese)

Nakamura, H. (1987). Hyperlipemia. Nihon Ishikai Zasshi 98 (Suppl), 203-207. (in Japanese) 